\title{
A multicenter study of oral health behavior among adult subjects from three South American cities
}

\author{
Mariel Viviana GÓMEZ (a) \\ Andrés TOLEDO (a) \\ Paola CARVAJAL (b) \\ Sabrina Carvalho GOMES (c) \\ Ricardo Santos Araújo COSTA (c) \\ Fernando SOLANES (b) \\ Rui Vicente OPPERMANN ${ }^{\text {(c) }}$ \\ Cassiano Kuchenbecker RÖSING (c) \\ Jorge GAMONAL (b) \\ Hugo ROMANELLI (a)
}

\footnotetext{
(a) Universidad Maimonides, School of Dentistry, Department of Periodontics, Buenos Aires, Argentina.

(b) Universidad de Chile, School of Dentistry, Department of Conservative Dentistry, Santiago, Chile.

(c) Universidade Federal do Rio Grande do Sul - UFRGS, School of Dentistry, Department of Conservative Dentistry, Porto Alegre, RS, Brazil.
}

Declaration of Interest: The authors certify that they have no commercial or associative interest that represents a conflict of interest in connection with the manuscript.

\section{Corresponding Author:}

Mariel Gómez,

E-mail:marielgomez@live.com

Submitted: August 09, 2017

Accepted for publication: January 26, 2018

Last revision: February 16, 2018

\begin{abstract}
The aims of this study were to describe the self-reported oral hygiene habits, dental visit frequency, and gingival bleeding perception in adult populations from three South American cities, and also to assess the association of these variables with sociodemographic data and with the clinical presence of plaque and gingival inflammation. Five-hundred and fifty adult subjects from each city (Porto Alegre, Brazil; Tucumán, Argentina; Santiago, Chile) received full mouth examinations to determine visible plaque and gingival index. A structured questionnaire on demographics, habits, attitudes and knowledge of oral health was also administered. The data were analyzed according to dental visit frequency, toothbrushing frequency, interproximal tooth cleaning frequency, subjects' perception of gum bleeding, and proportion of subject sites with VP and bleeding sites. Analysis of the association among the variables was performed using either a chi-square test or Fischer's exact test. Toothbrushing twice a day or more was reported by $84.2 \%$ of the subjects, but only $17.7 \%$ reported daily interdental cleaning, and $60.2 \%$ reported visiting a dental clinic only in an emergency. Only $2.97 \%$ had no bleeding sites, whereas $33.7 \%$ had $50 \%$ or more bleeding sites. Regular interdental self-cleaning and a dental visit every 3-6 months was associated with less plaque and less gingival bleeding. More than 12 years of education was associated with healthier habits, less bleeding and plaque scores. In conclusion, the oral health behavior of South American adult subjects from these cities is below the international recommendations, especially in relation to interdental cleaning and regular dental visits.
\end{abstract}

Keywords: Oral Hygiene; Epidemiology; Gingivitis.

\section{Introduction}

Self-preventive measures for the mechanical removal of dental plaque by adequate toothbrushing and interdental cleaning have proved to be the most significant factors in preventing oral infection diseases. ${ }^{1}$ Observing regular dental visits for preventive dental examinations, prophylaxis and professional reinforcement in oral hygiene instruction seems to be the key behavior for preventing these diseases..$^{2,3}$ Most oral problems, especially cavities and gingivitis, result from poor oral hygiene, and 
oral health status has been found to significantly affect general health and quality of life. ${ }^{4}$

Numerous surveys have been conducted in different populations worldwide to evaluate oral health behavior in adult subjects. Self-reported toothbrushing frequency, interdental cleaning and regular intervals between dental visits have been evaluated among adult samples from Europe, $, 5,6,7,8,9,10$ North America, ${ }^{11,12}$ Africa ${ }^{13}$ and Asia. ${ }^{14,15,16,17,18,19}$ Although data from these publications show great variability among countries, the association of the reported data with demographic information has shown differences ${ }^{20}$ according to gender, education, socioeconomic status and place of residence (urban or rural).

In Latin America, epidemiological data regarding oral health behavior are scarce, ${ }^{21}$ and disclose only limited information on adult subjects. Furthermore, the studies are either limited to students, ${ }^{22,23}$ teenagers ${ }^{24}$ or patients, ${ }^{25}$ or were conducted more than 15 years ago. ${ }^{26}$ In this sense, studies performed in South America are of foremost interest.

On the other hand, awareness of the signs of gingival disease is important in helping individuals seek treatment in the initial stages of the disease. Self-reported gingival bleeding is a symptom that has shown low sensitivity (0.35) for a threshold of $\geq 40 \%$ of bleeding sites in a Scottish sample of adult patients, ${ }^{27}$ and 0.42 for a threshold of $\geq 50 \%$ of bleeding sites in Swedish adults. ${ }^{28}$ Such data indicate that many people with clinically detected signs of gingival inflammation were unaware of any bleeding after toothbrushing. The perception of gingival bleeding has not yet been reported among South American populations.

Understanding regional behavior and the oral care compliance rates by the population can guide local public health practitioners and clinicians in designing and promoting oral health programs. Accordingly, we decided to describe the oral hygiene habits, the frequency of dental visits and the perception of gingival bleeding, as reported in a study sample of adults from three South American cities, and to assess the association of these variables with sociodemographic data and with the presence of plaque and gingival bleeding.

\section{Methodology}

\section{Study design}

The present study reports the findings of a multicenter population-based, cross-sectional epidemiological study conducted between January and July 2014

\section{Study population}

Study subjects were drawn from a noninstitutionalized adult population from three South American cities (Porto Alegre, Brazil; Tucumán, Argentina; and Santiago, Chile).

The study protocol followed the World Medical Association Declaration of Helsinki (version 2008) and was reviewed and approved by the Institutional Review Boards of the University of Chile, the Federal University of Rio Grande do Sul (Brazil) and the University of Maimónides (Argentina). All study participants were informed about the aims of the research and signed the informed consent form.

\section{Sample}

According to the inclusion criteria, the study participants had to (a) be 18 years of age or older, (b) have at least four permanent teeth, (c) not be at risk for bacterial endocarditis, (d) not be pregnant or lactating, (e) not be taking medication affecting the periodontal tissues, such as nonsteroidal anti-inflammatory drugs, calcium channel blockers, cyclosporine A or phenytoin, (f) not be taking anticoagulants, (g) not have fixed orthodontic appliances, and (h) be willing to sign a written consent form.

\section{Sampling and sample sizes}

A total of 1650 study subjects were evaluated (550 individuals per city). Based on an estimated prevalence of $93.9 \%$ on the gingival index $\geq 0.5,{ }^{29}$ the sample size that was needed to obtain a precision rate of $95 \%$ (confidence interval: $95 \%$ ) with a $2 \%$ range of error was estimated, and the adult participants in the study were selected using a multi-staged probability sample in order to achieve representativeness, as reported elsewhere. ${ }^{30}$ 


\section{Definition of variables}

\section{Oral health behavioral data and}

sociodemographic data

A structured questionnaire comprised of open and closed questions on demographics (gender, age, educational level and city), habits related to gingival health (toothbrushing frequency, use of interdental cleaning devices and dental visit frequency) and gum bleeding perception (do your gums bleed?) was administered to all the study subjects. The questionnaire was adapted to allow understanding among the three cities surveyed, and was tested in focus groups. ${ }^{30}$

The data were analyzed according to the following categories: a) age ( $<30$ or $\geq 30$ years of age); b) educational level ( $\leq 12$ or $>12$ years of school); c) dental visit frequency (every 3 months; every 6 months; every 12 months; only "when I have a problem"); d) toothbrushing frequency (<once a day; once a day; twice a day; >three times a day); g) interproximal tooth cleaning frequency (do not clean; < once a day; once a day; > once a day); and $h$ ) perception of gum bleeding (yes/no).

\section{Clinical data}

A clinical examination was performed on all the study subjects to evaluate the gingival inde ${ }^{31}$ and the presence/absence of visible plaque, ${ }^{32}$ as described below. The data obtained were analyzed according to the following categories: a) Gingival bleeding: number and percentage of subjects with no bleeding sites; $<10 \%$ of bleeding sites; between $\geq 10 \%$ and $<50 \%$ of bleeding sites; and $\geq 50 \%$ of bleeding sites; and b) Visible plaque: number and proportion of subjects with $\leq 20 \%$ of sites with visible plaque; between $20 \%$ and $\leq 50 \%$ of sites with visible plaque; and $>50 \%$ of sites with visible plaque.

Clinical examinations: All examinations were conducted by one calibrated examiner in each city. All the study subjects were evaluated for dental plaque and gingival inflammation. After applying water spray followed by gentle air drying and isolation with cotton rolls, six sites per tooth were evaluated (three facial and three lingual surfaces) in a full mouth examination conducted by a trained and calibrated examiner.

The examiners were trained in standardized diagnostic criteria and calibrated with the reference examiner (CKR). Each of the three examiners in the field (RC, AT, FS) conducted test examinations on at least 10 patients, and intra- and interexaminer kappa coefficients for the visible plaque index and gingival index were above 0.7 for all the examiners. Procedures were also performed to standardize the application of the structured questionnaire.

Visual plaque assessment: Absence (0) or presence (1) of dental plaque was scored for each site after visual examination. Gingival index: The Löe \& Silness Gingival Index system ${ }^{31}$ was used to evaluate gingival health. The examiners performed the gingival evaluations with gauze, compressed air, intraoral lighting, an oral mirror, a periodontal probe and a \#17 explorer.

Third molars and teeth with cervical restorations or prosthetic crowns were excluded from the scoring procedure. At the end of each day, the data were properly identified and coded, and then sent to the clinical investigator, who recorded the data. Approximately 15 examinations were performed per day.

\section{Statistical methods and data assessment}

Descriptive statistics were employed to calculate the prevalence for all the variables by city, and for the total study subject sample. The analysis of the association among the variables in contingency tables was performed using the chi-square tests or the Fisher exact probability test, where appropriate. Sensitivity and specificity were calculated. Statistical analyses were performed using a statistical software package (Stata/IC 13.1 for Mac). The level of significance was set at $\mathrm{p}<0.05$.

\section{Results}

A total of 1650 adults from the cities of Santiago, Tucumán and Porto Alegre completed the questionnaire and were examined. The distribution of the study sample according to age and gender is shown in Table 1.

Oral health behaviors (as self-reported by study subjects) and their relation to age, gender, educational level and city are shown in Table 2 - Toothbrushing 
Table 1. Study population/sample by city, age, and gender. $\mathrm{n}=550$.

\begin{tabular}{|c|c|c|c|c|c|c|c|}
\hline \multirow{3}{*}{ Age group } & All & \multicolumn{2}{|c|}{ Tucumán, Argentina } & \multicolumn{2}{|c|}{ Porto Alegre, Brazil } & \multicolumn{2}{|c|}{ Santiago, Chile } \\
\hline & \multirow{2}{*}{ n $(\%)$} & \multicolumn{2}{|c|}{ n (\%) } & \multicolumn{2}{|c|}{ n (\%) } & \multicolumn{2}{|c|}{ n (\%) } \\
\hline & & Male & Female & Male & Female & Male & Female \\
\hline 18-19 & $79(4.78)$ & $15(2.7)$ & $16(2.9)$ & $12(2.1)$ & $12(2.1)$ & $12(2.1)$ & $12(2.1)$ \\
\hline 20-29 & $378(22.90)$ & 73 (13.2) & $74(13.4)$ & $67(12.1)$ & $37(6.7)$ & $64(11.6)$ & $63(11.4)$ \\
\hline 30-39 & 348 (21.09) & 60 (10.9) & $66(12.0)$ & $57(10.3)$ & $62(11.2)$ & $52(9.4)$ & $51(9.2)$ \\
\hline $40-49$ & $300(18.18)$ & $41(7.4)$ & $50(9.09)$ & $49(8.9)$ & $58(10.5)$ & 49 (8.9) & $53(9.6)$ \\
\hline$\geq 50$ & $545(33.03)$ & 65 (1 1.8) & $90(16.3)$ & $85(15.4)$ & 111 (20.1) & 82 (14.9) & $112(20.3)$ \\
\hline Total & 1,65 & $254(46.1)$ & $296(53.8)$ & $270(49.0)$ & $280(50.9)$ & $259(47.0)$ & $291(52.9)$ \\
\hline
\end{tabular}

frequency: $84.2 \%$ of the study subjects reported that they brushed their teeth twice a day or more, and this was reported more frequently by women and by subjects with $>12$ years of education $(p=0.043)$. There were no age group differences in brushing frequency $(\mathrm{p}=0.629)$. Porto Alegre subjects reported that they brush twice a day or even more frequently $(p<0.001)$. Interdental cleaning frequency: Of the total study subject population, $66 \%$ reported that they do not clean interdentally, and only $17.7 \%$ clean daily. No difference was found between men and women $(p=0.287)$, but a significant association was found with age $(p=0.001)$ and level of education $(p<0.001)$. Study subjects $<30$ years of age, and subjects with $<12$ years of education reported a lower frequency of interdental cleaning. Interdental cleaning was significantly $(p<0.001)$ more frequent for Porto Alegre study subjects. Use of dental floss was more frequently reported (28.6\%) than toothpicks (2.4\%) and interdental brushes (2.4\%). Frequency of dental visits: The great majority of study subjects reported that they visit a dental clinic only in emergencies, and a higher percentage of study subjects with $>12$ years of education reported dental visits every 3 to 6 months ( $p<0.001$ ). No difference was found among gender or age group. A significant difference was found among the cities. Preventive dental visits made at least once a year were more frequent in subjects from Tucumán.

Plaque Index and Gingival Index (Table 3): Overall, $83.7 \%$ of the study subjects had plaque in $>50 \%$ of the sites in their mouth. Visible plaque was significantly associated with age, educational level and city of residence, and was more frequent in subjects $>30$ years of age $(p<0.001)$, with $\leq 12$ years of education $(p<0.001)$ and in subjects from Santiago ( $p<0.001)$. Bleeding in $\geq 50 \%$ of sites was a condition found in $33.7 \%$ of the subjects, and only $2.97 \%$ had no bleeding sites. Considering proximal sites, $96.3 \%$ of the study subjects had $\geq 50 \%$ of bleeding sites. Frequency of bleeding sites was significantly lower in females $(p=0.006)$, in younger subjects $(p=0.030)$, in subjects with $>12$ years of education $(\mathrm{p}<0.001)$, and in subjects from Tucumán and Porto Alegre $(\mathrm{p}<0.001)$.

Analysis of oral health habits and their association with plaque presence and gingival bleeding is shown in Tables 4 and 5. Study subjects who reported dental visits every 3 to 6 months had a lower percentage of bleeding sites, and less visible plaque $(p<0.001)$. The study subjects who reported brushing twice a day or more had a lower percentage of bleeding sites $(p=0.021)$. Regular use of interdental devices was associated with less plaque and with fewer bleeding sites $(\mathrm{p}<0.001)$.

Perception of gum bleeding (Table 6): $51.8 \%$ of the study subjects answered "yes" to the question "Do your gums bleed?" Subjects who answered positively to the question "Do your gums bleed?" had a higher proportion of sites with bleeding, compared with those who answered negatively, but $33 \%$ of the study subjects who reported no gum bleeding had $\geq 50 \%$ of bleeding sites. Positive answers were more frequently reported by younger study subjects $(p<0.001)$, by subjects with less education $(p=0.028)$ and by subjects from Porto Alegre $(p<0.001)$. 
Table 2. Self-reported oral health habits according to gender, age, educational level and city.

\begin{tabular}{|c|c|c|c|c|c|c|c|c|c|c|c|}
\hline \multirow[b]{2}{*}{ Variable } & \multicolumn{2}{|c|}{ Total subjects } & \multicolumn{2}{|c|}{ Gender - n (\%) } & \multicolumn{2}{|c|}{ Age - n (\%) } & \multicolumn{2}{|c|}{ Educational level - n (\%) } & \multicolumn{3}{|c|}{ City - n (\%) } \\
\hline & n (\%) & $95 \% \mathrm{Cl}$ & Female & Male & $<30$ & $\geq 30$ & $\leq 12$ & $>12$ & Tucumán & Santiago & $\begin{array}{l}\text { Porto } \\
\text { Alegre }\end{array}$ \\
\hline \multicolumn{12}{|l|}{ Dental visits } \\
\hline Every 3 month & $111(6.8)$ & $5.6-8.2$ & $60(7.0)$ & $51(6.6)$ & $34(7.7)$ & $77(6.5)$ & $71(6.6)$ & $40(7.3)$ & $66(12.5)$ & $14(2.5)$ & $31(5.7)$ \\
\hline Every 6 months & s $176(10.8)$ & 9.4-12.4 & $103(12.1)$ & $73(9.5)$ & $41(9.3)$ & $135(11.4)$ & $90(8.4)$ & $86(15.6)$ & $68(12.9)$ & $40(7.2)$ & $68(12.5)$ \\
\hline Every year & $356(22.0)$ & $20.0-24.1$ & $199(23.3)$ & $157(20.5)$ & $96(21.8)$ & $260(22.0)$ & $200(18.6)$ & $156(28.4)$ & $143(27.1)$ & $131(23.8)$ & $82(15.1)$ \\
\hline Emergency & $975(60.2)$ & $59.8-62.6$ & $491(57.5)$ & $484(63.2)$ & $268(61.0)$ & $707(59.9)$ & $709(66.2)$ & $266(48.5)$ & $250(47.4)$ & $364(66.3)$ & $361(66.6)$ \\
\hline No. of answers & 1,6 & & 853 & 765 & 439 & 1,179 & 548 & 1,07 & 527 & 549 & 542 \\
\hline$p$-value* & & & \multicolumn{2}{|c|}{0.188} & \multicolumn{2}{|c|}{0.559} & \multicolumn{2}{|c|}{0.000} & \multicolumn{3}{|c|}{0.000} \\
\hline \multicolumn{12}{|l|}{ Brushing } \\
\hline$<$ once/day & $57(3.5)$ & $2.6-4.5$ & $27(3.1)$ & $30(3.9)$ & $13(2.8)$ & $44(3.7)$ & $44(4.0)$ & $13(2.3)$ & $22(4.1)$ & $27(4.9)$ & $8(1.4)$ \\
\hline \multicolumn{12}{|l|}{ Frequency } \\
\hline once day & 198 (12.2) & 10.6-13.9 & $97(11.4)$ & $101(13.1)$ & $60(13.3)$ & 138(11.7) & 137 (12.7) & $61(11.1)$ & 71 (13.2) & 84(13.3) & $43(8.0)$ \\
\hline twice/day & $688(42.4)$ & $40.0-44.8$ & $343(40.3)$ & $345(44.8)$ & 195(43.3) & $493(42.1)$ & $433(40.2)$ & $255(46.7)$ & $262(48.9)$ & $261(47.5)$ & $165(30.7)$ \\
\hline$\geq$ three/day & $678(41.8)$ & $39.4-44.2$ & $384(45.1)$ & $294(38.1)$ & $182(40.4)$ & ) 496 (42.3) & 461 (42.8) & $217(39.7)$ & $180(33.6)$ & $177(32.2)$ & $321(59.7)$ \\
\hline No. of answers & 1,6 & & 851 & 770 & 450 & 1,171 & 1,075 & 546 & 535 & 549 & 537 \\
\hline$p$-value* & & & \multicolumn{2}{|c|}{0.043} & \multicolumn{2}{|c|}{0.629} & \multicolumn{2}{|c|}{0.043} & \multicolumn{3}{|c|}{0.000} \\
\hline \multicolumn{12}{|l|}{ Interdental } \\
\hline No & $\begin{array}{c}1087 \\
(66.0)\end{array}$ & $63.6-68.2$ & $565(65.3)$ & $522(66.6)$ & $318(69.7)$ & $769(64.5)$ & $799(72.8)$ & $288(52.3)$ & $415(75.7)$ & $394(71.7)$ & $278(50.5)$ \\
\hline \multicolumn{12}{|l|}{ Cleaning } \\
\hline Floss & $472(28.6)$ & 26.4-30.9 & $256(29.6)$ & $216(27.5)$ & $120(26.3)$ & $352(29.5)$ & $255(23.2)$ & $217(39.45)$ & $80(14.6)$ & $140(25.5)$ & $252(45.8)$ \\
\hline Brush & $41(2.4)$ & 1.7-3.3 & $21(2.4)$ & $20(2.5)$ & $10(2.1)$ & $31(2.6)$ & 15(1.3) & $26(4.7)$ & $28(5.1)$ & $9(1.6)$ & $4(0.7)$ \\
\hline Stick & $41(2.4)$ & 1.7-3.3 & $17(1.9)$ & $24(3.0)$ & $8(1.7)$ & $33(2.7)$ & $23(2.1)$ & $18(3.2)$ & $24(4.3)$ & $2(0.3)$ & $15(2.7)$ \\
\hline Other & $6(0.3)$ & $0.0-0.0$ & $5(0.5)$ & $1(0.1)$ & 0 & $6(0.5)$ & $5(0.4)$ & $1(0.1)$ & $1(0.1)$ & $4(0.7)$ & $1(0.1)$ \\
\hline No. of answers & 1,6 & 647 & 864 & 783 & 456 & 1,191 & 1,097 & 550 & 548 & 549 & 550 \\
\hline$p$-value* & & & \multicolumn{2}{|c|}{0.287} & \multicolumn{2}{|c|}{0.169} & \multicolumn{2}{|c|}{0.000} & \multicolumn{3}{|c|}{0.000} \\
\hline \multicolumn{12}{|c|}{ Interdental cleaning frequency } \\
\hline No use & $\begin{array}{c}1085 \\
(66.8)\end{array}$ & $63.7-68.3$ & $567(66.0)$ & $518(66.1)$ & $317(69.8)$ & $768(64.6)$ & $796(72.8)$ & $289(52.6)$ & $411(75.5)$ & $396(72.2)$ & $278(50.5)$ \\
\hline$<$ once/day & $266(16.2)$ & $14.4-18.0$ & $129(15.0)$ & $137(17.5)$ & $79(17.4)$ & $187(15.7)$ & $141(12.9)$ & $125(22.7)$ & 73(13.4) & $67(12.2)$ & $126(22.9)$ \\
\hline once/day & $194(11.8)$ & $10.2-13.4$ & $105(12.2)$ & 89(11.3) & $47(10.3)$ & $147(12.3)$ & $97(8.8)$ & $97(17.6)$ & $49(9.0)$ & $63(11.5)$ & $82(14.9)$ \\
\hline$>$ once/ day & $97(5.9)$ & 4.8-7.1 & $58(6.7)$ & $39(4.9)$ & $11(2,4)$ & $86(7.2)$ & $59(5.4)$ & $38(6.9)$ & $11(2.0)$ & $22(4.0)$ & $64(11.6)$ \\
\hline No. of answers & 1,6 & & 859 & 783 & 454 & 1,188 & 1093 & 549 & 544 & 548 & 550 \\
\hline $\mathrm{p}$-value* & & & \multicolumn{2}{|c|}{0.263} & \multicolumn{2}{|c|}{0.001} & \multicolumn{2}{|c|}{0.000} & & 0.000 & \\
\hline
\end{tabular}

*p-value: Pearson Chi square test was used to assess differences between frequency of each variable and gender, age, educational level and city. 
A multicenter study of oral health behavior among adult subjects from three South American cities

Table 3. Distribution of subjects ( $\mathrm{n}$ and \%) according to frequency of bleeding sites $(G \mid 2+3)$ and frequency of visible plaque sites, for the total sample and all demographic categories.

\begin{tabular}{|c|c|c|c|c|c|c|c|c|c|c|c|}
\hline \multirow{3}{*}{ Clinical variable } & \multicolumn{2}{|c|}{ Total subjects } & \multicolumn{2}{|c|}{ Gender } & \multicolumn{2}{|c|}{ Age } & \multicolumn{2}{|c|}{ Educational level } & \multicolumn{3}{|c|}{ City } \\
\hline & n (\%) & $95 \% \mathrm{Cl}$ & Female & Male & $<30$ & $\geq 30$ & $\leq 12$ & $>12$ & Tucumán & Santiago & $\begin{array}{l}\text { Porto } \\
\text { Alegre }\end{array}$ \\
\hline & \multicolumn{2}{|c|}{1,65} & $867(52.5)$ & $783(47.4)$ & $457(27.7)$ & 1,193 (72.3) & $1,099(66.6)$ & $551(33.3)$ & $550(33.3)$ & $550(33.3)$ & $550(33.3)$ \\
\hline \multicolumn{12}{|l|}{ Bleeding sites (\%) } \\
\hline No bleeding & $49(2.9)$ & $2.2-3.9$ & $34(3.9)$ & 15 (1.9) & $15(3.28)$ & $34(2.85)$ & $24(2.1)$ & $25(4.5)$ & $31(5.6)$ & $3(0.5)$ & $15(2.7)$ \\
\hline$\leq 10 \%$ & $310(18.7)$ & 16.9-20.7 & $142(16.3)$ & $168(21.4)$ & $99(21.6)$ & $211(17.6)$ & $200(18.2)$ & 110 (19.9) & $159(28.9)$ & $7(1.2)$ & $144(26.1)$ \\
\hline$>10 \%-<50 \%$ & $734(44.4)$ & $42.0-46.9$ & $400(46.1)$ & $334(42.6)$ & $213(46.6)$ & $521(43.6)$ & $532(48.4)$ & $202(36.6)$ & $306(55.6)$ & $89(16.1)$ & $339(61.6)$ \\
\hline$\geq 50 \%$ & $557(33.7)$ & $31.4-36.0$ & $291(33.4)$ & $266(33.9)$ & $130(28.4)$ & $427(35.7)$ & $343(31.2)$ & $214(38.8)$ & $54(9.8)$ & $451(82.0)$ & $52(9.4)$ \\
\hline$p$-value* & & & \multicolumn{2}{|c|}{0.006} & \multicolumn{2}{|c|}{0.030} & \multicolumn{2}{|c|}{0.000} & \multicolumn{3}{|c|}{0.000} \\
\hline \multicolumn{12}{|c|}{ Sites with visible plaque (\%) } \\
\hline$\leq 20 \%$ & $22(1.3)$ & $0.8-2.0$ & $13(1.5)$ & $9(1.1)$ & $8(1.7)$ & $14(1.1)$ & $8(0.7)$ & $14(2.54)$ & $13(2.3)$ & $2(0.36)$ & $7(1.2)$ \\
\hline$>20 \%-\leq 50 \%$ & $246(14.9)$ & $13.2-16.7$ & $113(13.0)$ & $133(16.9)$ & $103(22.5)$ & ) 143 (11.9) & $11(10.1)$ & $135(24.5)$ & $116(21.0)$ & $50(9.0)$ & $80(14.55)$ \\
\hline$>50 \%$ & $1,382(83.7)$ & $81.8-85.5$ & $741(85.4)$ & $641(81.8)$ & $346(75.7)$ & $1,036(86.8)$ & $980(89.1)$ & $402(72.9)$ & $421(76.5)$ & $498(90.5)$ & $463(84.1)$ \\
\hline $\mathrm{p}$-value * & & & \multicolumn{2}{|c|}{0.070} & \multicolumn{2}{|c|}{0.000} & \multicolumn{2}{|c|}{0.000} & \multicolumn{3}{|c|}{0.000} \\
\hline \multicolumn{12}{|c|}{ Interproximal sites with visible plaque (\%) } \\
\hline$\leq 20 \%$ & $17(1.0)$ & $0.6-1.6$ & $11(1.2)$ & $6(0.7)$ & $7(1.5)$ & $10(0.8)$ & $7(0.6)$ & $10(1.8)$ & $12(2.1)$ & $1(0.1)$ & $4(0,7)$ \\
\hline$>20 \%-\leq 50 \%$ & $175(10.6)$ & $9.1-21.1$ & $82(9.4)$ & 93 (1 1.8) & $67(14.6)$ & $108(9.0)$ & $76(6.9)$ & 99 (17.9) & $113(20.5)$ & $23(4.1)$ & $39(7.0)$ \\
\hline$>50 \%$ & 1,458 (88.3) & $86.1-89.1$ & 774 (89.2) & 684 (87.3) & $383(83.8)$ & $1,075(90.1)$ & $1,016(92.4)$ & $442(80.2)$ & 425 (77.2) & $526(95.6)$ & 507 (92.1) \\
\hline $\mathrm{p}$-value* & & & \multicolumn{2}{|c|}{0.184} & \multicolumn{2}{|c|}{0.002} & \multicolumn{2}{|c|}{0.000} & \multicolumn{3}{|c|}{0.000} \\
\hline
\end{tabular}

Gl: gingival index; ${ }^{*}$-value: Pearson Chi square test was used to assess differences between frequency of each variable and gender, age, educational level and city.

Table 4. Oral health behavior variables by prevalence of visible interproximal plaque: distribution of subjects (n and \%) according to frequency of visible plaque sites.

\begin{tabular}{|c|c|c|c|c|c|}
\hline Variable & Total subjects & $\begin{array}{l}\text { Subjects with } \leq 20 \% \\
\text { interproximal sites with } \\
\text { visible plaque }\end{array}$ & $\begin{array}{c}\text { Subjects with }>20 \%-\leq \\
50 \% \text { interproximal sites } \\
\text { with visible plaque }\end{array}$ & $\begin{array}{c}\text { Subjects with }>50 \% \\
\text { sites with visible plaque } \\
\text { interproximal }\end{array}$ & $p$-value* \\
\hline \multicolumn{6}{|l|}{ Dental visits } \\
\hline Every 3-6 months & $287(17.7)$ & $6(2.0)$ & $53(18.4)$ & $228(79.4)$ & \multirow{3}{*}{0.000} \\
\hline Every year & $356(22.0)$ & $6(1.6)$ & $53(14.8)$ & $297(83.4)$ & \\
\hline Emergency & $975(60.2)$ & $5(0.5)$ & $68(6.9)$ & $902(92.5)$ & \\
\hline \multicolumn{6}{|l|}{ Brushing frequency } \\
\hline$\leq$ once / day & $268(16.4)$ & $4(1.4)$ & $20(7.4)$ & $244(91.0)$ & \multirow{2}{*}{0.154} \\
\hline$\geq$ twice / day & $1,366(83.6)$ & $13(0.9)$ & $152(11.1)$ & $1,201(87.9)$ & \\
\hline \multicolumn{6}{|l|}{ Interdental cleaning } \\
\hline Yes & $560(34.0)$ & $9(1.6)$ & $84(15.0)$ & $467(83.3)$ & \multirow{2}{*}{0.000} \\
\hline No & $1,087(66.0)$ & $8(0.6)$ & $91(8.3)$ & $988(90.8)$ & \\
\hline \multicolumn{6}{|c|}{ Interdental cleaning frequency } \\
\hline$\geq$ once / day & $291(17.7)$ & $6(2.0)$ & $40(1.3)$ & $245(84.1)$ & \multirow{2}{*}{0.020} \\
\hline$<$ once / day & 1,351 (83.2) & $11(0.8)$ & $134(9.9)$ & 1,206 (89.2) & \\
\hline
\end{tabular}

${ }^{*} p$-value: Pearson Chi square test was used to assess differences between frequency of each oral health habit variable and visible plaque frequency. 
Table 5. Oral health behavior variables by prevalence of gingival bleeding: Distribution of subjects (n and \%) according to bleeding site frequency (GI 2+3).

\begin{tabular}{|c|c|c|c|c|c|c|}
\hline Variable & Total subjects & $\begin{array}{c}\text { Subjects without } \\
\text { bleeding sites }\end{array}$ & $\begin{array}{c}\text { Subjects with }<10 \% \\
\text { bleeding sites }\end{array}$ & $\begin{array}{c}\text { Subjects with } \geq 10 \\
<50 \% \text { bleeding } \\
\text { sites }\end{array}$ & $\begin{array}{l}\text { Subjects with } \geq \\
50 \% \text { bleeding sites }\end{array}$ & $\mathrm{p}$-value* \\
\hline \multicolumn{7}{|l|}{ Dental visits } \\
\hline Every 3-6 months & $287(17.7)$ & $14(4.8)$ & $69(24.0)$ & $148(51.5)$ & $56(19.5)$ & \multirow{3}{*}{0.000} \\
\hline Every year & $356(22.0)$ & $16(4.4)$ & 78 (21.9) & $146(41.0)$ & $116(32.5)$ & \\
\hline Emergency & $975(60.2)$ & $19(0,0)$ & $157(16.1)$ & $421(43.1)$ & $378(38.7)$ & \\
\hline \multicolumn{7}{|l|}{ Brushing frequency } \\
\hline$\leq$ once / day & $268(16.4)$ & $6(2.2)$ & $43(16.0)$ & $106(39.5)$ & $113(42.1)$ & \multirow{2}{*}{0.021} \\
\hline$\geq$ twice / day & 1,366 (83.6) & $43(3.1)$ & $263(19.2)$ & $620(45.3)$ & $440(32.2)$ & \\
\hline \multicolumn{7}{|l|}{ Interdental cleaning } \\
\hline Yes & $560(34.0)$ & $25(4.4)$ & $132(23.5)$ & $273(48.7)$ & $130(23.3)$ & \multirow{2}{*}{0.000} \\
\hline No & 1087 (66.0) & $24(2.2)$ & $177(16.2)$ & $460(42.3)$ & $426(39.1)$ & \\
\hline \multicolumn{7}{|c|}{ Interdental cleaning frequency } \\
\hline$\geq$ once / day & $291(17.7)$ & $16(5.5)$ & $68(23.3)$ & $134(46.0)$ & $73(25.0)$ & \multirow{2}{*}{0.000} \\
\hline$<$ once / day & 1,351 (83.2) & $33(2.4)$ & $240(17.7)$ & $595(44,0)$ & $483(35.7)$ & \\
\hline
\end{tabular}

The agreement between perception and clinical examination was evaluated by sensitivity and specificity (Table 7). A cross-tabulation shows that $51.5 \%$ of the study subjects $(286 / 555)$ having $\geq 50 \%$ clinical bleeding sites perceived bleeding as positive ("yes"), i.e., the sensitivity of the question was 0.51 , whereas $48 \%$ of the participants (525/1093) who had $<50 \%$ of clinical bleeding did not perceive the bleeding as positive ("no"), i.e. the specificity was 0.48 .

\section{Discussion}

This paper describes dental self-care practices among a large study sample of urban South American adult subjects. Three South American cities were chosen to be part of this investigation: Santiago, the capital of Chile, comprising 5.6 million inhabitants; Tucumán, the fifth largest city in Argentina, with 1.6 million inhabitants; and Porto Alegre, in southern Brazil, comprising approximately 1.5 million inhabitants. The choice of the cities was based on the possibility of logistics, taking into consideration that large cities, with at least 1 million inhabitants, better express the data distribution in highly populated areas. The analytical approach is the core of this study, which made the associations meaningful.
Table 6. Self-reported gum bleeding according to gender, age, city, educational level and \% bleeding sites per subject.

\begin{tabular}{|c|c|c|c|}
\hline \multirow{2}{*}{ Variable } & \multicolumn{2}{|c|}{ Self-reported gum bleeding $\mathrm{n}(\%)$} & \multirow{2}{*}{$p$-value* } \\
\hline & No & Yes & \\
\hline \multicolumn{4}{|l|}{ Gender } \\
\hline Female & $418(48.2)$ & $448(51.7)$ & \multirow{2}{*}{0.940} \\
\hline Male & $376(48)$ & $406(51.9)$ & \\
\hline \multicolumn{4}{|l|}{ Age } \\
\hline$<30$ & $169(36.9)$ & $288(63.0)$ & \multirow{2}{*}{0.000} \\
\hline$\geq 30$ & $625(52.4)$ & $566(47.5)$ & \\
\hline \multicolumn{4}{|l|}{ City } \\
\hline Santiago & $293(53.4)$ & $255(46.5)$ & \multirow{3}{*}{0.000} \\
\hline Tucumán & $276(50.1)$ & $274(49.8)$ & \\
\hline Porto Alegre & $225(40.9)$ & $325(59.0)$ & \\
\hline \multicolumn{4}{|c|}{ Educational level } \\
\hline$\leq 12$ years & $508(46.2)$ & $590(53.7)$ & \multirow{2}{*}{0.028} \\
\hline$>12$ years & $286(52.0)$ & $264(48.0)$ & \\
\hline \multicolumn{4}{|c|}{ Bleeding sites (\%) } \\
\hline No bleeding & $40(5.0)$ & $9(2.9)$ & \multirow{5}{*}{0.000} \\
\hline$<10$ & $178(22.4)$ & $132(18.8)$ & \\
\hline$\geq 10<50$ & $307(38.8)$ & $427(50.0)$ & \\
\hline$\geq 50$ & $269(33.8)$ & $286(33.4)$ & \\
\hline Total & $794(48.1)$ & $854(51.8)$ & \\
\hline
\end{tabular}

${ }^{*} p$-value: Pearson Chi square test was used to assess differences between frequency for each variable and self-reported gingival bleeding. 
Table 7. Self-reported gum bleeding and percentage of bleeding sites (threshold $\geq 50 \%$ ).

\begin{tabular}{lccc}
\hline $\begin{array}{l}\text { Clinical bleeding } \\
\text { sites (\%) }\end{array}$ & $\begin{array}{c}\text { No. of subjects } \\
\text { in each bleeding } \\
\text { category }\end{array}$ & $\begin{array}{c}\text { Self-reported gum } \\
\text { bleeding? }\end{array}$ \\
\cline { 3 - 4 }$<50 \%$ & 1,093 & 525 & 568 \\
$\geq 50 \%$ & 555 & 269 & 286 \\
Total no. of subjects & 1,648 & 794 & 854 \\
Sensitivity - $\mathrm{n}(95 \% \mathrm{Cl})$ & $0.51(0.47-0.54)$ & \\
Specificity - $\mathrm{n}(95 \% \mathrm{Cl})$ & $0.48(0.44-0.51)$ & \\
\hline
\end{tabular}

Overall, our results reveal that although $84.2 \%$ of the participants reported brushing twice a day or more, $66 \%$ never clean interproximally, and only $39.6 \%$ seek a preventive dental checkup at least once a year. In our study sample, a great number of subjects had plaque on more than $50 \%$ of their dental surfaces, and had bleeding on proximal sites.

Men reported brushing their teeth slightly less frequently than women, but no other gender differences were found in regard to the other behavioral variables studied. Clinically, no bleeding was a condition more frequent in women. Younger subjects reported less frequent use of interdental devices, and older subjects had more surfaces with plaque and more gingival bleeding. More years of education was significantly associated with higher frequency of toothbrushing, use of dental floss, regular dental checkups, less gingival bleeding and lower plaque scores. Interestingly, variations were found among the cities, that is, dental visits were more frequent among Tucumán study subjects, and interdental cleaning was more frequent among Porto Alegre subjects. These differences in behavior probably explain why study subjects from Santiago presented the highest levels of plaque and bleeding sites. ${ }^{30}$

Our findings on oral health habits agree with those of other studies conducted in Latin America; a high proportion of subjects reported brushing twice a day or more, but a lower proportion reported cleaning interproximally. In a study of university students $<35$ years of age from Colombia ${ }^{22}$, whereas $94 \%$ of the study subjects brushed their teeth twice daily, only $49 \%$ used dental floss. In Argentina, of the 3,694 patients who visited general dentists, $72.2 \%$ reported brushing twice a day, but only $38 \%$ used dental floss ${ }^{25}$. In a group of 471 study subjects, 24 to 44 years of age from Porto Alegre, ${ }^{26} 95 \%$ reported brushing twice a day or more, and $67.6 \%$ reported using dental floss. In our sample from Porto Alegre, $50 \%$ of the study subjects reported using interdental devices, a greater proportion than the near 30\% reported from Tucumán and Santiago. These differences probably reflect different public health policies among the cities.

A comparison of data from urban populations shows variations in the brushing frequency reported from country to country, but there is agreement that daily interdental cleaning is always less frequent than daily toothbrushing. Oral health habits similar to our findings were reported in Denmark, ${ }^{7}$ Canada $^{11}$ and Australia. ${ }^{33} \mathrm{~A}$ higher frequency of interdental cleaning was reported in a middle-aged sample of university employees from Lithuanian, ${ }^{10}$ where $55 \%$ reported using interdental cleaning tools on a daily basis. Remarkably poorer oral health behavior for both toothbrushing and interdental cleaning has been reported in samples from Iran ${ }_{1}^{19}$ Kuwait, ${ }^{14}$ Nigeria, ${ }^{13}$ China $^{17}$ and Saudi Arabia. ${ }^{15}$

Although data from various publications show great variability, our findings corroborate those of numerous previousstudies reporting that better oral hygiene practices are significantly related to education, $7,12,13,14,26,33$ and that toothbrushing frequency is related to gender, ${ }^{710,13,14,15,17,1,22,26}$ In agreement with some studies, ${ }^{14,34}$ we found no gender difference in interdental cleaning frequency, although other studies found that interdental cleaning was more frequently reported by women.,17,19,26,33

Previous studies that have evaluated the effect of age on oral health habits are difficult to compare with because of the variability in the age groups considered, but there is a general trend that older subjects use extra cleaning devices more frequently. $18,26,33$

In relation to dental visits, at least one visit a year was reported by only $39 \%$ of our study subjects. This proportion is lower than that reported in some developed counties. In a Canadian sample, $69 \%$ of the study subjects had yearly dental examinations, ${ }^{11} 69.9 \%$ in a United States sample, ${ }^{35} 58.5 \%$ in an Australian sample, ${ }^{33}$ and $51 \%$ of the respondents from Lithuanian, ${ }^{10}$ 
whereas almost $90 \%$ from Sweden ${ }^{9}$ claimed having regular dental checkups. A lower frequency of dental visits has been reported in Nigeria, ${ }^{13}$ where only $26.4 \%$ of the subjects visited the dentist at least once a year, and in China, ${ }^{17}$ where only $28 \%$ of the subjects reported visiting a dentist in the previous 12 months.

In an adult sample from the United States, ${ }^{34}$ a statistical relationship was found among years of education, yearly checkups, dental insurance and income. In our study, years of education and city of residence were associated with the frequency of dental checkups. The highest frequency of dental visits found among Tucumán residents is likely related to a government policy that encourages yearly dental checkups at no cost to the patients.

In regard to the relationship between oral health behavior and gingival health, we observed that low frequency of interdental cleaning and no regular preventative dental care were associated with the presence of more plaque and gingival inflammation, as has been reported in previous studies. ${ }^{33,34}$ In relation to toothbrushing and gingival health, toothbrushing twice daily or more among our study subjects was associated with a lower proportion of gingival bleeding sites per subject. In relation to this result, Crocombe et al. ${ }^{33}$ in the Australian survey found that higher brushing frequency was associated with less dental plaque and gingivitis in a bivariate analysis, but only with less plaque in a multivariate analysis.

The correlation between self-assessment of gum bleeding and clinical findings showed a sensitivity of 0.51 , that is, half of the subjects with clinically positive bleeding perceived bleeding, and the other half did not. Low sensitivity values were also reported by Buhlin et al. ${ }^{28}$ and Gilbert and Nuttall, ${ }^{27}$ who found sensitivities of 0.42 and 0.35 , respectively. These results demonstrate that many subjects with clinically detectable gingival bleeding were unaware of any

\section{References}

1. Lang NP, Cumming BR, Löe H. Toothbrushing frequency as it relates to plaque development and gingival health. J Periodontol. 1973 Jul;44 (7):396-405. https://doi.org/10.1902/jop.1973.44.7.396 bleeding after toothbrushing or flossing; therefore, the self-reporting of these signs may underestimate the actual manifestation of the disease. The use of measurements that enable accuracy in calculating is a matter of interest and is part of the validation process of any diagnostic tool. We understand that self-reported perceptions of oral conditions are being made with greater awareness, and always need to be tested. Since these are patient-centered outcomes, it is always important to report these self-reported values, which need to be further investigated in the case of the present study.

One possible limitation of the present study is that the independent variables were collected from self-reported information. However, this is the most common way of gathering answers of this kind, even though they could be biased, because they rely on memory. Nevertheless, this is a recognized survey tool in epidemiology.

\section{Conclusions}

The present study shows that the behavior of South American adult subjects from the cities of Santiago, Porto Alegre and Tucumán, in regard to their oral health care practices, is below the international recommendations, especially with regard to interdental cleaning and regular dental care visits.

Toothbrushing frequency, interdental cleaning and regular preventive dental care are important indicators of gingival health, and educational level is by far the most significant determinant of good oral health behavior.

\section{Acknowledgements}

The authors declare no conflict of interest. This study received the support of the ColgatePalmolive Company.

\footnotetext{
2. Axelsson P, Nyström B, Lindhe J. The long-term effect of a plaque control program on tooth mortality, caries and periodontal disease in adults: results after 30 years of maintenance. J Clin Periodontol. 2004 Sep;31 (9):749-57. https://doi.org/10.1111/i.1600-051X.2004.00563.x
} 
3. Armitage GC, Xenoudi P. Post-treatment supportive care for the natural dentition and dental implants. Periodontol 2000. 2016 Jun;71 (1):164-84. https://doi.org/10.1111/prd.12122

4. Locker D, Quiñonez C. To what extent do oral disorders compromise the quality of life? Community Dent Oral Epidemiol. 2011 Feb;39 (1):3-11. https://doi.org/10.1111/j.1600-0528.2010.00597.x

5. Petersen PE, Aleksejuniene J, Christensen LB, Eriksen HM, Kalo I. Oral health behavior and attitudes of adults in Lithuania. Acta Odontol Scand. 2000 Dec;58 (6):243-8. https://doi.org/10.1080/00016350050217073

6. Bradnock G, White DA, Nuttall NM, Morris AJ, Treasure ET, Pine CM. Dental attitudes and behaviours in 1998 and implications for the future. Br Dent J. 2001 Mar;190 (5):22832. https://doi.org/10.1038/si.bdj.4800935a

7. Christensen LB, Petersen PE, Krustrup U, Kjøller M. Selfreported oral hygiene practices among adults in Denmark. Community Dent Health. 2003 Dec;20 (4):229-35.

8. Suominen-Taipale L, Nordblad A, Vehkalahti M, Aromaa A, editors. Oral health in the Finnish adult population: health 2000 survey. Helsinki: KTL-National Public Health Institute; 2008.

9. Molarius A, Engström S, Flink H, Simonsson B, Tegelberg A. Socioeconomic differences in self-rated oral health and dental care utilisation after the dental care reform in 2008 in Sweden. BMC Oral Health. 2014 Nov;14 (1):134. https://doi.org/10.1186/1472-6831-14-134

10. Sakalauskienė Z, Vehkalahti M, Murtomaa H, Mačiulskienė V. Factors related to gender differences in toothbrushing among Lithuanian middle-aged university employees. Medicina (Kaunas). 2011;47 (3):180-6.

11. Payne BJ, Locker D. Relationship between dental and general health behaviors in a Canadian population. J Public Health Dent. 1996;56 (4):198-204. https://doi.org/10.1111/j.1752-7325.1996.tb02436.x

12. Ronis DL, Lang WP, Farghaly MM, Passow E. Tooth brushing, flossing, and preventive dental visits by Detroit-area residents in relation to demographic and socioeconomic factors. J Public Health Dent. 1993;53 (3):138-45. https://doi.org/10.1111/j.1752-7325.1993.tb02692.x

13. Olusile AO, Adeniyi AA, Orebanjo O. Self-rated oral health status, oral health service utilization, and oral hygiene practices among adult Nigerians. BMC Oral Health. 2014 Nov;14 (1):140. https://doi.org/10.1186/1472-6831-14-140

14. Al-Shammari KF, Al-Ansari JM, Al-Khabbaz AK, Dashti A, Honkala EJ. Self-reported oral hygiene habits and oral health problems of Kuwaiti adults. Med Princ Pract. 2007;16 (1):15-21. https://doi.org/10.1159/000096134

15. Al-Otaibi M, Zimmerman M, Angmar-Månsson B. Prevailing oral hygiene practices among urban Saudi Arabians in relation to age, gender and socio-economic background. Acta Odontol Scand. 2003 Aug;61 (4):212-6. https://doi.org/10.1080/00016350310004070

16. Kumar TS, Dagli RJ, Mathur A, Jain M, Balasubramanyam $G$, Prabu $D$ et al. Oral health status and practices of dentate
Bhil adult tribes of southern Rajasthan, India. Int Dent J. 2009 Jun;59 (3):133-40.

17. Zhu L, Petersen PE, Wang HY, Bian JY, Zhang BX. Oral health knowledge, attitudes and behaviour of adults in China. Int Dent J. 2005 Aug;55 (4):231-41. https://doi.org/10.1111/j.1875-595X.2005.tb00321.x

18. Harada S, Akhter R, Kurita K, Mori M, Hoshikoshi M, Tamashiro $\mathrm{H}$ et al. Relationships between lifestyle and dental health behaviors in a rural population in Japan. Community Dent Oral Epidemiol. 2005 Feb;33 (1):17-24. https://doi.org/10.1111/j.1600-0528.2004.00189.x

19. Asgari F, Majidi A, Koohpayehzadeh J, Etemad K, Rafei A. Oral hygiene status in a general population of Iran, 2011: a key lifestyle marker in relation to common risk factors of non-communicable diseases. Int J Health Policy Manag. 2015 Jan;4 (6):343-52. https://doi.org/10.15171/ijhpm.2015.18

20. Sakki TK, Knuuttila ML, Anttila SS. Lifestyle, gender and occupational status as determinants of dental health behavior. J Clin Periodontol. 1998 Jul;25 (7):566-70. https://doi.org/10.1111/i.1600-051X.1998.tb02489.x

21. Oppermann R, Haas A, Kuchenbecker Rosing C, Susin C. Epidemiology of periodontal diseases in adults from Latin America. Periodontology 2000. 2015 Feb;67 (1):13-33. https://doi.org/10.1111/prd.12061

22. Marulanda A, Coral D, Sabogal D, Serrano C. Periodontal conditions of Colombian university students aged 16 to 35. Braz Oral Res. 2014 May;28 (5):1-7. https://doi. org/10.1590/1807-3107BOR-2014.vol28.0009

23. Espinoza Santander I, Muñoz PobleteC, Lara Molina M, Uribe Cifuentes JP. Hábitos de higiene oral en estudiantes de odontología de la Universidad de Chile. Rev Clín Periodoncia Implantol Rehabil Oral. 2010 Apr;3 (1):11-8. https://doi.org/10.1016/S0718-5391 (10)70035-5

24. Freddo SL, Aerts DR, Abegg C, Davoglio R, Vieira PC, Monteiro L. [Oral hygiene habits and use of dental services among teenage students in a city in southern Brazil]. Cad Saude Publica. 2008 Sep;24 (9):1991-2000. https://doi.org/10.1590/S0102-311X2008000900005

25. Romanelli H, Gonzalez y Rivas M, Chiappe V, Gómez M, Macchi R. Periodontal treatment needs in Argentine adult subjects. Acta Odontol Latinoam. 2007;20 (1):39-47.

26. Abegg C. [Oral hygiene habits among Brazilian adults in an urban area of Southern Brazil]. Rev Saúde Pública. 1997 Dec;31 (6):586-93. Portuguese. https://doi.org/10.1590/S0034-89101997000700007

27. Gilbert $A D$, Nuttall NM. Self-reporting of periodontal health status. Br Dent J. 1999 Mar;186 (5):241-4.

28. Buhlin K, Gustafsson A, Andersson K, Håkansson J, Klinge B. Validity and limitations of self-reported periodontal health. Community Dent Oral Epidemiol. 2002 Dec;30 (6):431-7. https://doi.org/10.1034/j.1600-0528.2002.00014.x

29. Li Y, Lee S, Hujoel P, Su M, Zhang W, Kim J et al. Prevalence and severity of gingivitis in American adults. Am J Dent. 2010 Feb;23 (1):9-13. 
30. Carvajal P, Gómez M, Gomes S, Costa R, Toledo A, Solanes $\mathrm{F}$ et al. Prevalence, severity, and risk indicators of gingival inflammation in a multi-center study on South American adults: a cross sectional study. J Appl Oral Sci. 2016 Sep-Oct;24 (5):524-34. https://doi.org/10.1590/1678-775720160178

31. Löe H. The Gingival Index, the Plaque Index and the Retention Index Systems. J Periodontol 1967 Nov-Dec; 38 (6):Suppl 610-6. https://doi.org/10.1902/jop.1967.38.6.610

32. Ainamo J, Bay I. Problems and proposals for recording gingivitis and plaque. Int Dent J. 1975 Dec;25 (4):229-35.

33. Crocombe LA, Brennan DS, Slade GD, Loc DO. Is self interdental cleaning associated with dental plaque levels, dental calculus, gingivitis and periodontal disease? J Periodontal Res. 2012 Apr;47 (2):188-97. https://doi.org/10.1111/j.1600-0765.2011.01420.x 34. Lang WP, Farghaly MM, Ronis DL. The relation of preventive dental behaviors to periodontal health status. J Clin Periodontol. 1994 Mar;21 (3):194-8. https://doi.org/10.1111/j.1600-051X.1994.tb00303.x

35. Akinkugbe A, Lucas-Perry E. Trends in dental visits among the US non-institutionalized civilian population: findings from BRFSS 1995-2008. J Theory Pract Dent Public Health. 2013; (2):32-6. 